\title{
Assistive Robot Arm Controlled by a P300-based Brain Machine Interface for Daily Activities
}

\author{
David Achanccaray*, Juan M. Chau*, Jairo Pirca, Francisco Sepulveda, and Mitsuhiro Hayashibe
}

\begin{abstract}
This work proposes an assistive system for everyday activities composed by a brain machine interface (BMI) based on P300 to choose a predefined task, a robot arm to perform the chosen task, and a stereo vision subsystem developed with two cameras for object recognition and coordinates calculation. The system was tested with eight healthy subjects; its results were greater BMI accuracies, lower 3D coordinates calculation error, and lower task execution time than similar systems. However, it should be tested with disabled subjects to provide more reliable end-user results. Regardless, this system is suitable to assist healthy subjects for performing reaching task to grasp objects in daily activities, and the intuitive interface would be useful for disabled subjects.
\end{abstract}

\section{INTRODUCTION}

Stroke is one of the most important causes of global mortality, the number of stroke deaths increased in $40 \%$ in the last two decades [1]. It was reported in 2016 as the second cause of death by The World Health Organization (WHO) Global Health Estimates, and it was also the second leading cause of disability-adjusted life years (DALY) [2]. Mainly, the disability consists of partial or total paralysis, often of the upper limbs; and around $80 \%$ of post stroke patients resulting in hemiparesis [3].

Research in rehabilitation for patients with paralysis due to stroke or severe damage in the central nervous system has had a significant progress in the last years, but it is not enough. Rehabilitation therapies still require an intense labor from therapists, and motivation and effort from patients; leading to long periods of time before noticeable recovery [4]. Complementary assistive technology could be used to help patients to do everyday tasks and recover autonomy partially.

Typical peripheral devices to assist patients are wheelchairs. orthosis, and prosthesis; and the interface between users and peripherals is built with mechanical and/or

This work was supported by FONDECYT from CONCYTEC, Peru. Contract 112-2017 and project grant J004-2016.

*The authors contributed equally to this work.

David Achanccaray is with the Neuro-Robotics Laboratory, Department of Robotics, Tohoku University, Sendai, Japan (e-mail: david.ad@dc.tohoku.ac.jp). He is also with the Biomechanics and Applied Robotics Research Group, Department of Engineering, Pontifical Catholic University of Peru, Lima, Peru.

Juan M. Chau and Jairo Pirca are with the Biomechanics and Applied Robotics Research Group, Department of Engineering, Pontifical Catholic University of Peru, Lima, Peru (e-mail: jmchau@pucp.edu.pe, pirca.jairo@pucp.pe).

Francisco Sepulveda is with the Brain Computer Interfaces and Neural Engineering Research Group, University of Essex, Colchester, UK (e-mail: f.sepulveda@essex.ac.uk).

Mitsuhiro Hayashibe is with the Neuro-Robotics Laboratory, Department of Robotics, Tohoku University, Sendai, Japan (e-mail: hayashibe@tohoku.ac.jp). electrical subsystems [4]. Currently, there are more complex assistive devices like exoskeletons, smart prosthesis, and wearable or non-wearable robots based on inertial measurements or physiological signals to control them; such as electromyography (EMG) and electroencephalography (EEG) [5].

A brain machine interface (BMI) is based on EEG signals; it is able to recognize brain activity patterns, and it allows direct operation of devices despite the user's physical disabilities [4]. BMIs are currently being used to control assistive robots for disable people, but a framework of this kind of system usually involves shared control between the BMI and the robot; i.e., the user makes a choice through a BMI about the movements or tasks to be performed by the robot, while the robot has an internal control system to perform these actions [5].

A wheelchair was controlled by a BMI based on motor imagery (MI) [6], it also comprises shared control, and computer vision for obstacle detection. Power band features from power spectral density (PSD) in mu band are extracted, then a Gaussian classifier is trained to recognize PSD changes during MI tasks; the classifier mean accuracy was 95\% for four healthy subjects. According to these results, this approach gives to the user greater flexibility and spontaneous interaction than similar systems based on P300 paradigm, P300 is the positive response that occurs 300 milliseconds after an oddball stimulus. P300 and MI paradigm were combined in [7] to operate a wheelchair with shared control in familiar environments, where P300 waves are used for predefined destination selection and MI tasks are used as a stop option. A support vector machine (SVM) classifier was trained for P300 detection, and the mean accuracy for five healthy subjects was around $100 \%$. Although, it is not possible to compare mental workload for both studies, each of them shows advantages for a specific case.

An architecture to operate an assistive robot based on a BMI based on P300 is approached in [8]; this system is composed by a BMI to select a command for the robot, an assistive Kinova Jaco robot arm to execute commands, and a Microsoft Kinect One for object recognition and user's face detection. The P300-based BMI uses the software system BCI2000, and to train a Stepwise Linear Discriminant Analysis (SWLDA) for P300 wave detection. In their study, the classifier accuracy is not mentioned as its approach, it is based on robot operability and motion control, having a time for task execution of at least $70 \mathrm{~s}$; nevertheless, it shows a high potential of application for upper limb disabled people.

MI-based BMI to control a robotic arm has been tested with tetraplegics $[9,10]$, where shared control is carried out by an internal robot control subsystem and an eye tracking 
module to define trajectories; a Gaussian classifier was trained and its mean accuracy for nine patients was about $81 \%$. Most of patients reported to feel discomfort during the experiments; however, only three patients had the robot movement as a feedback, and they felt that they were controlling it, giving them a sense of agency. The rest of patients observed a movement cursor as in any typical brain computer interface (BCI) experiment.

Another similar BMI system was based on Steady-State Visual Evoked Potential (SSVEP) [11], SSVEP paradigm consists in stimuli flashing at different frequencies are associated to specific commands, this system consisted of a BMI to choose a predefined task or control directly the robot's movements, a robot arm to perform the chosen task or movements, and a stereo camera for object recognition. This BMI 's mean accuracy is not mentioned, as its focus was on the system speed and architecture; although, the mean time of four healthy subjects for task selection was $9.34 \mathrm{~s}$. Furthermore, the system's performance was evaluated on four disabled subjects, they felt that the system functionalities would be able to improve their quality of life, but not enough to replace care-giving personnel.

Usually, SSVEP is used for a BMI with few options (until 6 approximately) and P300 is used for a BMI with many options (at least 4); P300-based BMI shows a greater accuracy than SSVEP-based BMI, however it depends on the number of options and the number of repetitions of P300 stimuli [12]. P300 was chosen for our system due to be more reliable if the number of options is increased.

In this work, a P300-based BMI to control an assistive robot arm for everyday activities is proposed; the system is developed based on shared control that includes a BMI to choose predefined tasks, an internal robot control module to perform the chosen task, and a stereo vision subsystem with two cameras for object recognition and coordinates calculation.

\section{MATERIALS AND METHODS}

\section{A. Participants}

Eight healthy subjects have participated in this project, aged between 20 and 28 years, with none of them having previous experience with BMIs. This study was conducted according to the guidelines of the Declaration of Helsinki. The participants signed an informed consent before to start the experiments.

\section{B. Experimental Setup}

The system used for this study is composed by a g.Nautilus (from g.tec) amplifier with dry active electrodes to record EEG data. The electrodes were distributed according to the $10 / 10$ International System, and they are placed in the positions FP1, FP2, F3, FZ, F4, T7, C3, CZ, C4, T8, CP5, CP6, P3, PZ, P4, OZ; using AFz as the ground electrode, and right mastoid as reference. EEG data is sampled at $500 \mathrm{~Hz}$. Additionally, two cameras Logitech C525 were mounted on a Kinova Mico robot arm; which has 6 degrees of freedom and supports a payload of $2.1 \mathrm{Kg}$, these cameras have a resolution of $2 \mathrm{MP}$ and visual field of $69^{\circ}$.

All devices were connected to a computer Intel Core i7-6500U at 2.5 GHz, 8 GB RAM, Nvidia GeForce 940MX

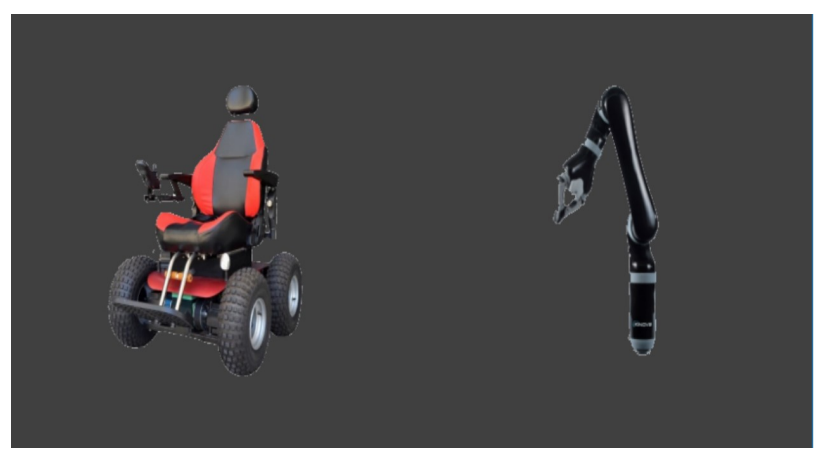

Figure 1. Images used as stimuli in BMI training stage.

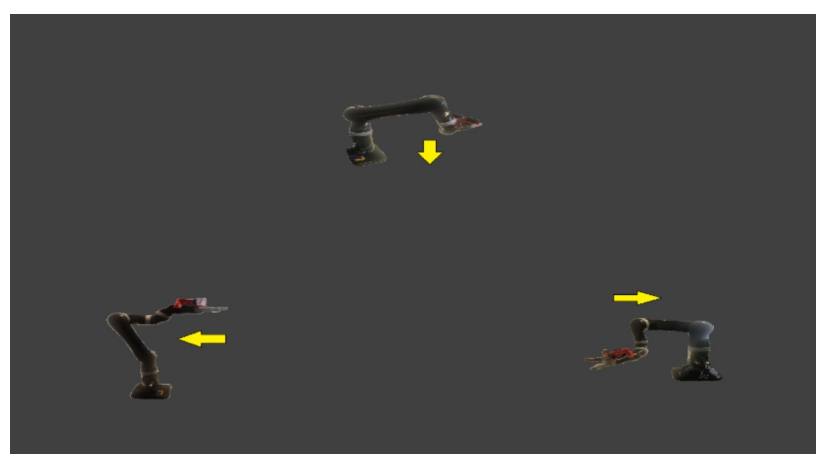

Figure 2. Images used as stimuli in the second level of BMI test stage.

a)

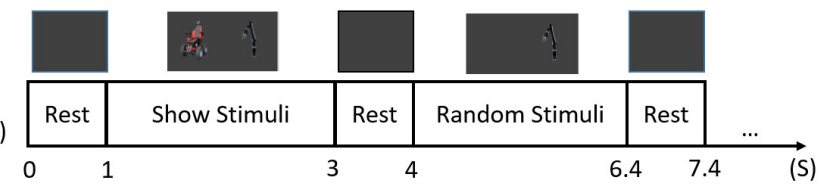

b)

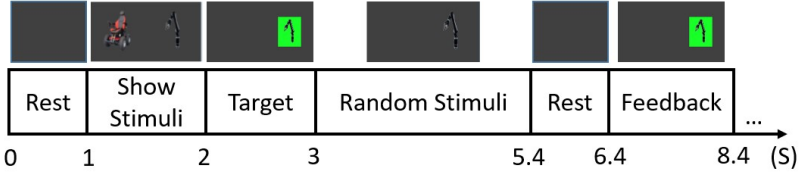

Figure 3. Timeline of a sequence. (a) Training stage. (b) Test stage.

GPU and Microsoft Windows 10 as operating system. Signal and Image processing are done using Matlab software (MathWorks, Inc).

\section{Experimental Procedure}

The protocol consisted of two stages, training and test; each stage is performed in different sessions. The first one is for EEG data acquisition to train the P300-based BMI and is composed of two sessions, where a one-level user interface with two stimuli is displayed to the user, a wheelchair and a robotic arm, see Figure 1; both stimuli are flashing randomly to evoke a P300 signal, if the user was focused in one of them. These stimuli correspond to two different peripherals, but only the robotic arm control was developed; the wheelchair control will be developed in future works.

The user was seated in front of a computer screen, while its EEG data was recorded; the stimuli were shown during 2 
seconds and random flashing stimuli were shown during 2.4 seconds by sequence, see Figure 3.a, each stimulus was shown for 100 milliseconds and hidden for 300 milliseconds during this time. Each trial was composed of 3 sequences, each sequence includes each stimulus once; and each run is composed of 6 trials; then, each session was composed of 6 runs.

The second stage was the test of the proposed system; a two-level user interface was shown to the user, with the first level being the same as the one used for the data acquisition and the second level as shown in the Figure 2, it is comprised of 3 options of robot arm predefined tasks. In this stage, the stimuli were displayed for 1 second, then random flashing stimuli were displayed for 2.4 seconds in the first level and 3.6 seconds in the second level, and feedback was provided for 2 seconds; see Figure 3.b. After feedback in the second level, a predefined task which could take up to 60 seconds to be performed was executed by the robot.

\section{Signal Processing}

EEG data was filtered by a 10th order Butterworth bandpass filter between 1 and $15 \mathrm{~Hz}$. Then, it was downsampled by a factor of 10 . Common spatial patterns (CSP) [13] was applied to the reduced EEG data using all the CSP filters; then, it was normalized by standard normalization.

The EEG data acquired and processed in the stage of data acquisition was used to train a Bayesian linear discriminant analysis (BLDA) classifier [14] for P300 occurrence detection.

\section{E. Robot Arm Control}

In Figure 2, three predefined tasks associated to images are shown as options; being the first one to pick up an object and move it to the left, the second one to pick up an object and move it to the right, and the last one to pick up an object and move it close to the user's face.

After an option was chosen by P300 occurrence detection using the BLDA classifier, the Kinova Mico robot arm should perform the predefined task; previously, the object is detected and its position is determined to set it as the robot desired position, then the robot achieves this position through an internal control module and picks up the object.

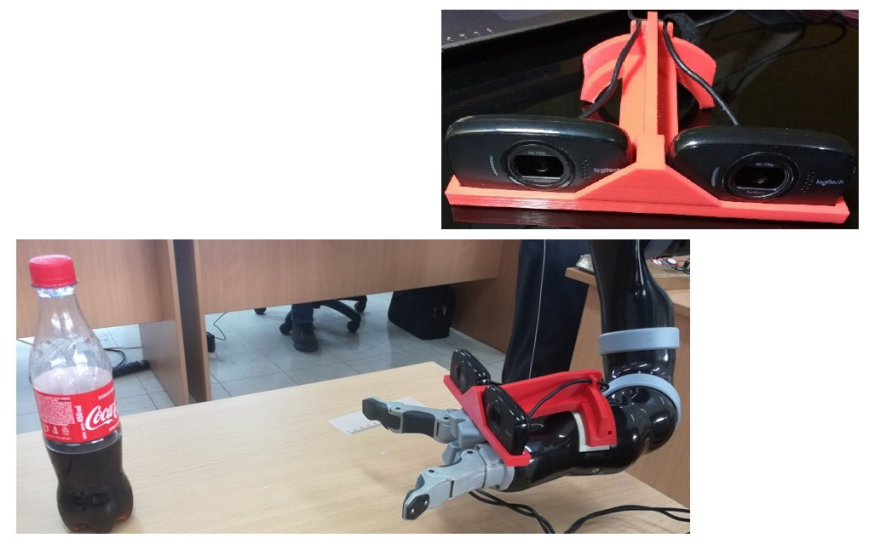

Figure 4. Stereo vision subsystem.

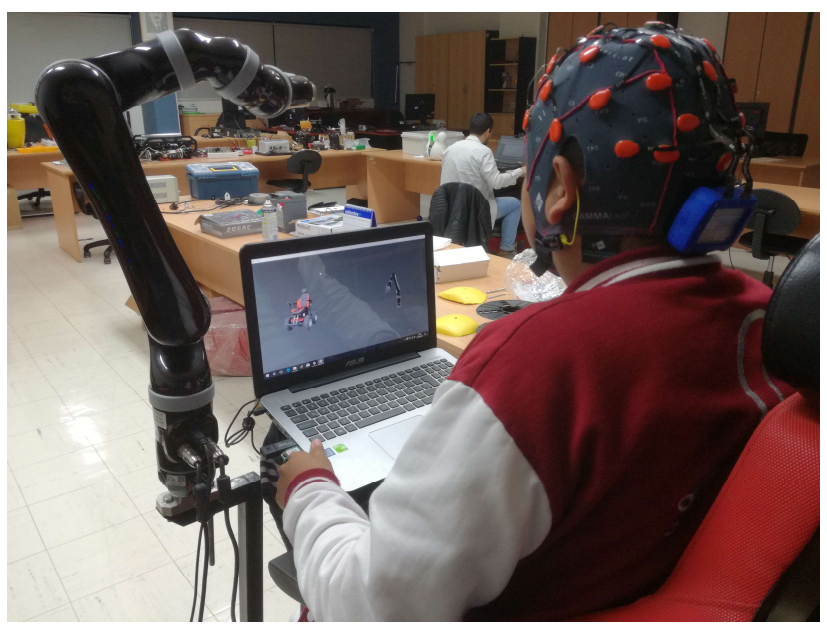

Figure 5. Participant testing the proposed assistive system.

The robot speed was calculated experimentally to have a smooth movement.

\section{F. Object Recognition and Coordinates Calculation}

A stereo vision subsystem was implemented with two cameras mounted over the robot arm, see Figure 4, to recognize predefined objects and calculate their 3D coordinates. The object recognition was based on the method scale-invariant feature transform (SIFT) to extract key points from images independently of its scale or orientation. 100 key points were chosen to recognize objects; then, these key points from both cameras were compared to identify common points and calculate its depth by triangulation using the distance between cameras.

Finally, 3D coordinates were calculated with the obtained measurements with respect to the coordinate system in the robot end-effector and they were transformed to the coordinate system in the robot base.

\section{RESULTS}

The Table I shows the accuracies of the P300-based BMI for training and test stages, the mean accuracies for both stages were $91.6 \%$ and $82.6 \%$ respectively. The P300 paradigm shows a high performance for the most of subjects with only three stimuli repetitions, despite of the BMI levels are comprised of two and three stimuli; it could mean lower training times for a P300-based BMI.

These accuracies were greater than the results in $[9,10]$; Despite that system was evaluated with disabled people, our system could have close results to the obtained ones if it was evaluated with disabled people. The Figure 5 shows a subject testing the proposed assistive system.

The time for task selection was constant, it is equal to $8.4 \mathrm{~s}$ for the first level and $9.6 \mathrm{~s}$ for the second level. This time is close to the task selection time mentioned in [11]; although, both paradigms are different, mental workload could be greater for SSVEP paradigm than for P300 paradigm.

The estimated mean depth error was around 4.75 millimeters $(\mathrm{mm})$ when the distance between objects and robot was in the range of 250 to $550 \mathrm{~mm}$. Then, the mean errors of $3 \mathrm{D}$ estimated coordinate for four different objects 
(PET bottle, can, deodorant spray, and small plastic bottle) with respect to the coordinates system in the robot base were $2.5 \mathrm{~mm}$ for the x-axis, $2 \mathrm{~mm}$ for the $\mathrm{y}$-axis, and $2.25 \mathrm{~mm}$ for the z-axis; in all cases, the robot arm was able to pick up the object regardless. The time for task execution was $60 \mathrm{~s}$, with the error and time being lower than the ones in [8].

Subjects were asked about how to stay focused on the stimuli and how the system should be improved. Most of subjects had no problem to keep focused on the chosen stimulus and it was not necessary to count the number of times each of them appeared as in [14]. Furthermore, subjects mentioned that the main improvements to the system should be performed on image quality and graphic design.

TABLE I. ACCURACIES OF THE P300 OCURRENCE DETECTION

\begin{tabular}{|c|r|r|}
\hline Subject & \multicolumn{1}{|c|}{ Training } & \multicolumn{1}{c|}{ Test } \\
\hline 1 & 100.0 & 100.0 \\
\hline 2 & 100.0 & 79.0 \\
\hline 3 & 85.0 & 70.0 \\
\hline 4 & 94.4 & 90.0 \\
\hline 5 & 75.6 & 70.0 \\
\hline 6 & 100.0 & 95.0 \\
\hline 7 & 94.4 & 95.0 \\
\hline 8 & 83.3 & 62.0 \\
\hline Mean & 91.6 & 82.6 \\
\hline
\end{tabular}

\section{CONCLUSIONS}

This work shows a P300-based BMI assistive system with a robot arm and a stereo vision subsystem for object detection and coordinates calculation to help disabled people to perform everyday activities. The system was tested with eight healthy subjects; with the BMI accuracies, the 3D coordinates calculation error, and the task execution time resulting better or close than the ones of similar systems.

However, the system has been tested only with healthy subjects; it also should be tested with disabled people to give more reliable end-user results. Furthermore, subjects should be asked about the robot movement perception, as the sense of ownership is an important factor to consider in order to have a more natural interface to control an assistive robotic system. We will further seek for the intuitive robot multi-joint arm control by benefiting synergetic computation for user to think only about the desired task [15].

Regardless, the proposed system shows a high performance assisting healthy subjects for performing reaching task to grasp objects in daily life environment, and the intuitive interface would be useful for disabled people.

\section{ACKNOWLEDGMENT}

The experiments were performed at the facilities of the Biomechanics and Robotics Research Laboratory (LIBRA) and the Manufacturing Advanced Technologies Center (CETAM), both laboratories belong to the Pontifical Catholic University of Peru.

\section{REFERENCES}

[1] E. J. Benjamin et al., "Heart disease and stroke statistics-2017 update: a report from the American Heart Association," Circulation, vol. 135, no. 10, pp. e146-e603, 2017.

[2] World Health Organization, "Global Health Estimates". [Online]. Available: http://www.who.int/healthinfo/global_burden_disease/en/

[3] I. Faria-Fortini, S. M. Michaelsen, J. G. Cassiano, and L. F. Teixeira-Salmela, "Upper extremity function in stroke subjects: relationships between the international classification of functioning, disability, and health domains," Journal of Hand Therapy, vol. 24, no. 3, pp. 257-265, 2011.

[4] Y. He, D. Eguren, J. M. Azorín, R. G. Grossman, T. P. Luu, and J. L. Contreras-Vidal, "Brain-machine interfaces for controlling lower-limb powered robotic systems," Journal of Neural Engineering, vol. 15, no. 2, pp. 021004, 2018.

[5] M. Tariq, P. M. Trivailo, and Milan Simic, "EEG-based BCI control schemes for lower-limb assistive-robots," Frontiers in Human Neuroscience, vol. 12, pp. 312, 2018

[6] T. Carlson and J. del R. Millan, "Brain-Controlled Wheelchairs: A Robotic Architecture," IEEE Robotics \& Automation Magazine, vol. 20, no. 1, pp. 65-73, March 2013.

[7] B. Rebsamen et al., "A Brain Controlled Wheelchair to Navigate in Familiar Environments," IEEE Transactions on Neural Systems and Rehabilitation Engineering, vol. 18, no. 6, pp. 590-598, Dec. 2010.

[8] F. Arrichiello, P. Di Lillo, D. Di Vito, G. Antonelli and S. Chiaverini, "Assistive robot operated via P300-based brain computer interface," 2017 IEEE International Conference on Robotics and Automation (ICRA), Singapore, 2017, pp. 6032-6037.

[9] M. Danóczy, S. Fazli, C. Grozea, K-R Müller, F. Popescu, "Brain2Robot: a grasping robot arm controlled by gaze and asynchronous EEG BCI," Proceedings of the Fourth International Brain-Computer Interface Workshop and Training Course, Graz, Austria, pp 355-360, 2008.

[10] G. Onose et al., "On the feasibility of using motor imagery EEG-based brain-computer interface in chronic tetraplegics for assistive robotic arm control: a clinical test and long-term post-trial follow-up," Spinal Cord, vol. 50, pp. 599-608, Aug. 2012.

[11] S. M. Grigorescu, T. Lüth, C. Fragkopoulos, M. Cyriacks, and A. Graeser, "A BCI-controlled robotic assistant for quadriplegic people in domestic and professional life," Robotica, New York: Cambridge University Press, vol. 30, pp. 419-431, 2012.

[12] J. Zhao, W. Li, M. Li, "Comparative Study of SSVEP- and P300-Based Models for the Telepresence Control of Humanoid Robots," Plos One, vol.10, pp. 1-18, Nov. 2015.

[13] D. Achanccaray, K. Pacheco, E. Carranza, and M. Hayashibe, "Immersive virtual reality feedback in a brain computer interface for upper limb rehabilitation," IEEE International Conference on Systems, Man, and Cybernetics (SMC), Miyazaki, 2018.

[14] U. Hoffmann, J. M. Vesin, T. Ebrahimi, and K. Diserens, "An efficient P300-based brain-computer interface for disabled subjects," Journal of Neuroscience Methods, vol. 167, no. 1, pp. 115-125, 2008.

[15] S. Bhattacharyya, S. Shimoda and M. Hayashibe, "A Synergetic Brain-Machine Interfacing Paradigm for Multi-DOF Robot Control," IEEE Transactions on Systems, Man, and Cybernetics: Systems, vol. 46, no. 7, pp. 957-968, July 2016. 